\title{
ORIGINAL
}

\section{The impact of frailty on ICU and 30-day mortality and the level of care in very elderly patients ( $\geq 80$ years)}

Hans Flaatten ${ }^{1,2^{*}} \mathbb{0}$, Dylan W. De Lange ${ }^{3}$, Alessandro Morandi4 ${ }^{4,5}$, Finn H. Andersen ${ }^{6,7}$, Antonio Artigas ${ }^{8}$, Guido Bertolini $^{10}$, Ariane Boumendil ${ }^{11}$, Maurizio Cecconi ${ }^{12}$, Steffen Christensen ${ }^{9}$, Loredana Faraldi ${ }^{13}$, Jesper Fjølner ${ }^{9}$, Christian Jung ${ }^{14}$, Brian Marsh ${ }^{15}$, Rui Moreno ${ }^{16}$, Sandra Oeyen ${ }^{17}$, Christina Agwald Öhman ${ }^{18}$, Bernardo Bollen Pinto ${ }^{19}$, Ivo W. Soliman ${ }^{20}$, Wojciech Szczeklik ${ }^{21}$, Andreas Valentin ${ }^{22}$, Ximena Watson ${ }^{12}$, Tilemachos Zaferidis ${ }^{23}$, Bertrand Guidet ${ }^{24,25,26}$ on behalf of the VIP1 study group

(C) 2017 Springer-Verlag GmbH Germany and ESICM

\begin{abstract}
Purpose: Very old critical ill patients are a rapid expanding group in the ICU. Indications for admission, triage criteria and level of care are frequently discussed for such patients. However, most relevant outcome studies in this group frequently find an increased mortality and a reduced quality of life in survivors. The main objective was to study the impact of frailty compared with other variables with regards to short-term outcome in the very old ICU population.

Methods: A transnational prospective cohort study from October 2016 to May 2017 with 30 days follow-up was set up by the European Society of Intensive Care Medicine. In total 311 ICUs from 21 European countries participated. The ICUs included the first consecutive 20 very old ( $\geq 80$ years) patients admitted to the ICU within a 3-month inclusion period. Frailty, SOFA score and therapeutic procedures were registered, in addition to limitations of care. For measurement of frailty the Clinical Frailty Scale was used at ICU admission. The main outcomes were ICU and 30-day mortality and survival at 30 days.
\end{abstract}

Results: A total of 5021 patients with a median age of 84 years (IQR 81-86 years) were included in the final analysis, 2404 (47.9\%) were women. Admission was classified as acute in 4215 (83.9\%) of the patients. Overall ICU and 30-day mortality rates were $22.1 \%$ and $32.6 \%$. During ICU stay $23.8 \%$ of the patients did not receive specific ICU procedures: ventilation, vasoactive drugs or renal replacement therapy. Frailty (values $\geq 5$ ) was found in $43.1 \%$ and was independently related to 30 -day survival (HR 1.54; $95 \% \mathrm{Cl} 1.38-1.73)$ for frail versus non-frail.

Conclusions: Among very old patients ( $\geq 80$ years) admitted to the ICU, the consecutive classes in Clinical Frailty Scale were inversely associated with short-term survival. The scale had a very low number of missing data. These findings provide support to add frailty to the clinical assessment in this patient group.

Trial registration: ClinicalTrials.gov (ID: NCT03134807).

\footnotetext{
*Correspondence: hans.flaatten@uib.no

${ }^{1}$ Department of Clinical Medicine, University of Bergen, Bergen, Norway

Full author information is available at the end of the article

The members of the VIP1 Study Group are listed in the

Acknowledgements and in the electronic supplementary material.
}

Take-home message: This prospective multinational study of 5021 very old intensive care patients ( $\geq 80$ years) found frailty to be present in $43.1 \%$ and it was independently related to ICU (22.1\%) and 30-day mortality (32.6\%).

\section{自 Springer}


Keywords: Octogenarians, Elderly, ICU, Mortality, Frailty, Severity of illness

\section{Introduction}

The increased demand for intensive care leaves many physicians with difficult decisions given the shortage of ICU beds in several countries. This is particularly true for "very old intensive care patients" (VIPs, $\geq 80$ years old) partly because their life expectancy is limited. Are ICU admission and treatment proportional to their chances of survival? Indeed, all European countries are faced with this growing challenge related to these VIPs.

The triage process prior to admitting VIPs to the ICU differs from the less old, and should ideally use different tools than in younger patients [1]. At present, we have no ideal combination of independent prognostic factors associated with benefit from intensive care in this group. Even within a country there may be different opinions about the triage process. Other variables, apart from age, are important prognostic factors in the critically ill elderly patient, like co-morbidity and acute organ failure. Geriatric syndromes like frailty, sarcopenia, delirium and dementia probably play a major role as well. Frailty has been defined as a clinical state of increased vulnerability from age-associated decline in physiological reserves and function in many physiological systems [2]. Although this concept of frailty is well established in the geriatric approach, it has only recently been used in ICU outcome studies [3].

Most publications on outcomes from intensive care in VIPs report a poor survival rate or a reduced quality of life in survivors [4-7], which is not merely dependent on the crude age of the patients. One-year mortality has been reported to reach $65 \%$ [8] and quality of life in survivors is significantly reduced in half of these survivors [4]. An international group of intensivists has recently proposed a research agenda for critically ill older patients [9]. Further documentation of the outcome of VIPs was deemed important, especially establishing the importance of factors like frailty and functional status.

The main objective of the present study was to study the occurrence of frailty and to assess its impact on 30-day mortality in patients 80 years of age or older admitted to European ICUs. A secondary objective was to report the intensity of care and treatment restrictions while on the ICU in this patient group.

\section{Methods}

\section{Design and setting}

We conducted a prospective multicentre study in several European countries. The study was coordinated through the Health Services Resource and Outcome
(HSRO) section of the European Society of Intensive Care Medicine (ESICM). The ESICM network was used to disseminate information about the study. In most participating European countries, dedicated national coordinators were responsible for recruitment of ICUs, coordinated national and local ethical permissions and supervised patient recruitment at the national level. Institutional research ethic board approval was obtained from each study site. Because of the diversity of ethical consent procedures in Europe, many countries could recruit patients without informed consent while some had to collect informed consent. Each participating ICU could choose to include consecutive patients during an entire 3-month period, or to include the first 20 consecutive patients within this period. Hence, units with many elderly patients could include more than 20 consecutive patients. Patients were followed until death or 30 days after ICU admission. Individual ICUs started the data collection between October 2016 and February 2017 depending on the speed of local ethical clearance. A website was set up to facilitate dissemination of information about the study and to allow for data entry using an electronic CRF. The week to week recruitment of patients at country level was displayed on the web page (ESM Fig. 1). The study was registered after start on ClinicalTrials.gov (ID: NCT03134807). No specific funding was received.

\section{Study population}

Consecutive patients of 80 years of age or older and admitted to the ICU were eligible, but pre-ICU triage was not registered. All reasons for ICU admissions were allowed and patients were grouped according to a predefined list of 12 admission groups: respiratory failure, circulatory failure, combined respiratory/circulatory failure, severe sepsis, severe trauma without head injury, severe trauma with head injury, isolated head injury, post elective surgery, intoxication, non-traumatic cerebral failure, post emergency surgery and other reasons. For the analysis in this paper patients were grouped into acute and elective (planned) admissions.

\section{Data collection}

The study collected a set of mandatory data (ESM Table 1) and allowed for optional registration of severity of illness scores. Length of stay (LOS) was recorded in hours, allowing calculation of length of stay in 24-h periods rather than in calendar days. Any period during the ICU stay on non-invasive or invasive ventilation with endotracheal intubation or tracheotomy, use of 
vasoactive drugs, and renal replacement therapy (RRT) qualified for use of that procedure/treatment. The SOFA score was calculated either manually or using an online calculator according to guidelines on admission [10].

The case record form and database ran on a secure server composed and stored in Aarhus University, Denmark. The servers were managed in co-operation between the Information Technology Department and the Department of Clinical Medicine.

\section{Frailty}

We defined this as the frailty level before the acute illness and hospital admission. The Clinical Frailty Scale (CFS) with simple visual description was used as a categorisation tool [11] and information necessary to perform this assessment by the ICU staff was given by patients or proxy. No particular training to use the CFS was deemed necessary since the description combined with illustrations is intuitive. The English version of the CSF was used for this study. The CSF is composed of nine classes from very fit to terminally ill; hence the last group is technically speaking not necessarily frail (ESM Fig. 2).

\section{Outcome measurement}

The main outcome was survival at ICU discharge and at 30 days after ICU admission. Data was retrieved from the hospital administration system in some units, but had to be collected using active follow-up in the rest. We also registered the use of four common ICU procedures during the ICU stay, but not their duration. Whether treatment was withheld or withdrawn was recorded and based on international recommendations [12].

\section{Statistical analysis}

No formal sample size calculation was performed for this observational study. Baseline patient characteristics, treatment and outcomes were compared between three frailty groups based on the CFS: not frail (scale 1-3), prefrail (scale 4) and frail (scale 5-9). Frailty was also analysed both using the whole scale as ordinal data, and after dichotomizing its presence, considering values of at least 5 as frail as used in previous publications [3].

Continuous variables were compared between groups using Mann-Whitney $U$ test and categorical variables were compared using the Chi square test. Normally distributed continuous data were described as means with 95\% CI and non-normal distributed data were described as median with $25-75$ percentiles. For these analysis SPSS (IBM SPSS statistics, version 24) was used.

The crude overall survival at 30 days was estimated by the Kaplan-Meier method and compared with the use of a log-rank test. The adjusted impact of frailty on 30-day survival was estimated using a Cox model. Variables included in the model were age, gender, SOFA score, type of ICU admission (elective versus acute). The analysis was conducted on acute admission only and with inclusion of geographical regions. A $p$ value of less than 0.05 was considered to indicate statistical significance. Analyses were performed with $\mathrm{R}$ software, version 3.2.2 (The $\mathrm{R}$ Foundation).

\section{Results}

In total, 311 ICUs from 21 European countries participated (Fig. 1 and ESM table of collaborators). In addition, three ICUs from outside Europe also provided data.

Overall 5187 patients had a CRF completed, while 5132 patients from Europe were included. Of European patients 5021 patients with a complete dataset could be analysed. Patient flow through the study is illustrated in the flow chart (ESM Fig. 3). The number of recruited patients varied from 1054 (UK 91 ICUs) to 6 (Czech Republic, one ICU). The median recruited patients per country was 114 and the median per ICU was 16.

Median age for all patients was 84 years (IQR 81-86) and $52.1 \%$ were male (ESM Table 2). Median LOS in hospital before ICU admission was 1 day, and median ICU LOS was 2.3 days. Overall survival was $77.9 \%$ in the ICU and $67.6 \%$ at 30 days. ICU survival was lower (72.4\%) in the subgroup of patients with an ICU LOS $<24 \mathrm{~h}$ (ESM Table 3). Patients dying within $24 \mathrm{~h}$ had very high SOFA score, and withholding or withdrawal of therapy was frequent (ESM Table 4).

Data on frailty was available in $99.8 \%$ of admissions with a median value of 4 (IQR 3-6). Differences between not-frail, pre-frail and frail groups are depicted in Table 1. Frail patients were older, had higher SOFA score, were more often female, and more frequently therapy was withheld or withdrawn. The crude 30 -day mortality for each frailty score (1-9) in elective and acute admitted patients is shown in Fig. 2 with nearly linear relationship between mortality and increased frailty in the acute admitted patients (Chi squared test for trend; $p<0.001$ ).

The distribution of the 12 admission categories is shown in ESM Table 5. Respiratory and/or circulatory failure were the most frequent causes. Baseline data and outcomes for the admission groups are given in Table 2, regrouped into acute and elective admissions. Patients admitted electively had less organ failure and less mortality at ICU discharge and at 30 days.

The most frequent ICU procedures were the use of vasoactive drugs in $52.2 \%$ and invasive ventilation in $50.7 \%$, NIV was used in $23 \%$ while RRT was used in $9.2 \%$ of all admissions. In $23.8 \%$ of the patients no ICU procedures were recorded.

Cox survival analysis (Table 3 ) gives the data on univariate and multivariate analysis with corresponding hazard 


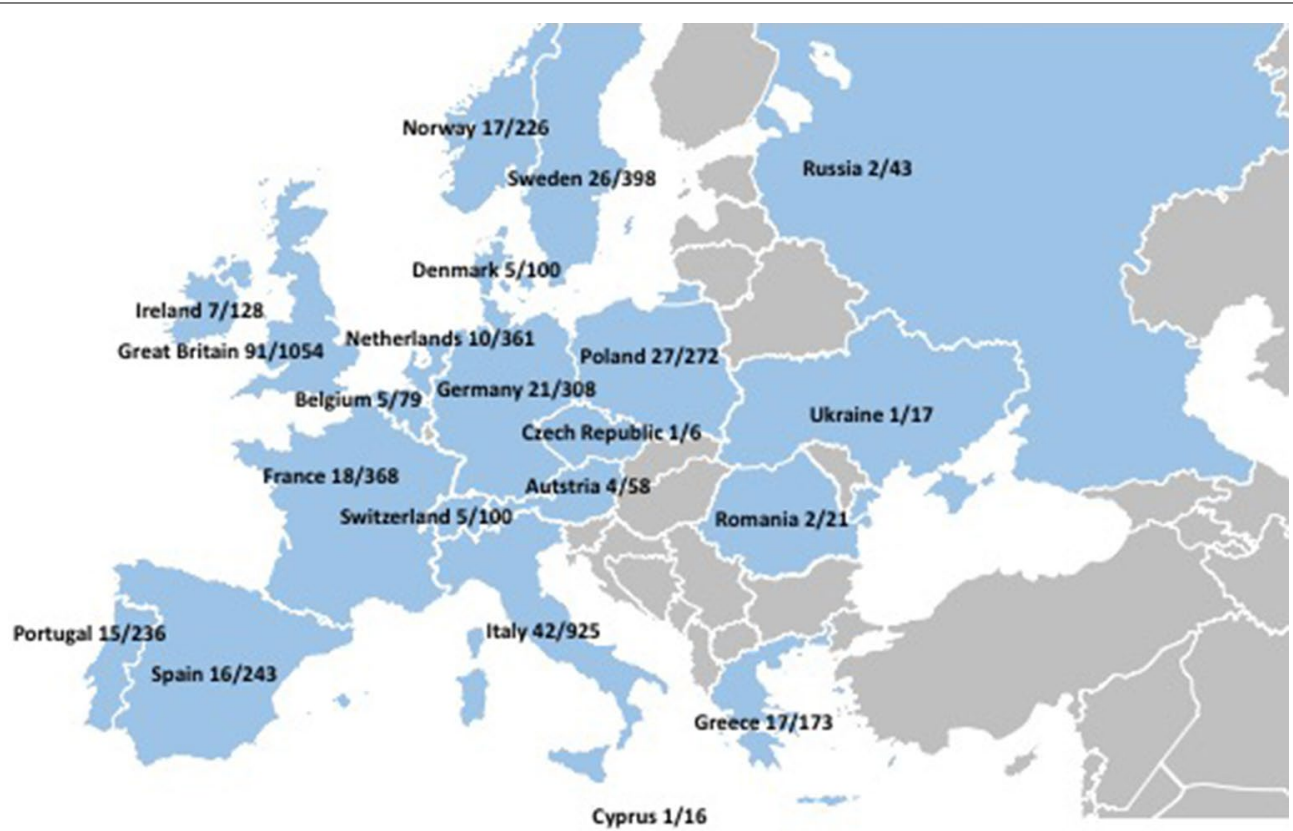

Fig. 1 Participating countries, ICU numbers and included patients

Table 1 Comparison of three classes of frailty: not frail, pre-frail and frail

\begin{tabular}{|c|c|c|c|c|}
\hline Frailty score group descriptives & Frailty 1-3: not frail & Frailty 4: pre-frail & Frailty 5-9: frail & $p$ value \\
\hline$N$ & 1893 & 972 & 2156 & \\
\hline Age & $83[81-86]$ & $83[81-86]$ & 84 [82-87] & $<0.001$ \\
\hline Gender (male) & $1100(58.1 \%)$ & $510(52.5 \%)$ & $1007(46.7 \%)$ & $<0.001$ \\
\hline Unplanned admission & $436(23 \%)$ & $211(21.7 \%)$ & $259(12 \%)$ & $<0.001$ \\
\hline SOFA score at admission & $6[3-9]$ & $6[4-10]$ & $7[4-11]$ & $<0.001$ \\
\hline Region & & & & $<0.001$ \\
\hline Central & $286(15.1 \%)$ & $174(17.9 \%)$ & $441(20.5 \%)$ & \\
\hline East & $133(7 \%)$ & $111(11.4 \%)$ & $303(14.1 \%)$ & \\
\hline North & $241(12.7 \%)$ & $131(13.5 \%)$ & $350(16.2 \%)$ & \\
\hline South & $734(38.8 \%)$ & $310(31.9 \%)$ & $658(30.5 \%)$ & \\
\hline West & $499(26.4 \%)$ & $246(25.3 \%)$ & $404(18.7 \%)$ & \\
\hline ICU length of stay (days) & $2.2[1.0-5.6]$ & $2.4[1.0-6.0]$ & $2.4[1.0-6.0]$ & 0.695 \\
\hline Treatment withheld & $269(14.2 \%)$ & $206(21.2 \%)$ & $674(31.3 \%)$ & $<0.001$ \\
\hline Treatment withdrawn & $187(9.9 \%)$ & $106(10.9 \%)$ & $319(14.8 \%)$ & $<0.001$ \\
\hline ICU survival & 1558 (82.3\%) & 775 (79.7\%) & 1578 (73.2\%) & $<0.001$ \\
\hline 30-day survival & $1431(75.6 \%)$ & $686(70.6 \%)$ & 1278 (59.3\%) & $<0.001$ \\
\hline
\end{tabular}

Data are presented as medians [interquartile range] or absolute numbers $N(\%)$

ratios (HR). The highest HRs were found for acute versus elective admissions (4.72) with frail versus fit as the second (1.54). The HR for age per 5-year increase was 1.2. The estimated 30-day survival rates for fit versus frail were $76 \%$ and $59 \%$, respectively. Results of a subgroup analysis of acute admitted patients gave very similar results (see ESM Table 6A), and results from including five different European regions in the analysis are shown in ESM Table 6B.

\section{Discussion}

Along with the increase in life expectancy of the general population, the age of critically ill patients increases. The proportion of very elderly ICU patients exceeds $10 \%$ in 


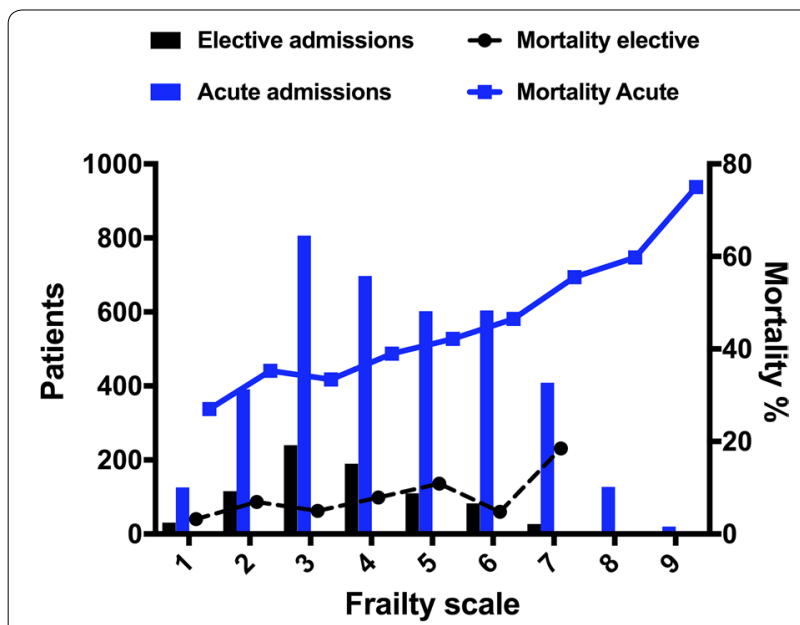

Fig. 2 Association between frailty scale and 30-day outcome

Table 2 Comparison of elective versus acute admissions

\begin{tabular}{lllr}
\hline Admission & Elective & Acute & $p$ value \\
\hline$N$ & 906 & 4115 & \\
Age & $83[81-86]$ & $84[81-87]$ & $<0.001$ \\
Gender male & $499(55.1 \%)$ & $2118(51.5 \%)$ & 0.054 \\
SOFA score at admission & $4[2-7]$ & $7[4-11]$ & $<0.001$ \\
Frailty scale & & & $<0.001$ \\
\hline Frailty 1-3: not frail & $436(48.1 \%)$ & $1457(35.4 \%)$ & \\
Frailty 4: pre-frail & $211(23.3 \%)$ & $761(18.5 \%)$ & \\
\hline Frailty 5-9: frail & $259(28.6 \%)$ & $1897(46.1 \%)$ & \\
Region & & & $<0.001$ \\
\hline Central & $241(26.6 \%)$ & $660(16 \%)$ & \\
East & $84(9.3 \%)$ & $463(11.3 \%)$ & \\
\hline North & $40(4.4 \%)$ & $682(16.6 \%)$ & \\
South & $257(28.4 \%)$ & $1445(35.1 \%)$ & \\
\hline West & $284(31.3 \%)$ & $865(21 \%)$ & \\
ICU length of stay (days) & $1.2[0.9-2.9]$ & $2.8[1.1-6.5]$ & $<0.001$ \\
\hline Treatment withheld & $42(4.6 \%)$ & $1107(26.9 \%)$ & $<0.001$ \\
\hline Treatment withdrawn & $15(1.7 \%)$ & $597(14.5 \%)$ & $<0.001$ \\
\hline ICU survival & $878(96.9 \%)$ & $3033(73.7 \%)$ & $<0.001$ \\
\hline 30-day survival & $844(93.2 \%)$ & $2551(62 \%)$ & $<0.001$ \\
\hline Data & & & \\
\hline
\end{tabular}

Data are presented as medians [interquartile range] or absolute numbers $N(\%)$

many countries. However, the survival of this very old age group is rather poor $[4,5,9]$. One of the important gaps of knowledge is the short-term survival of these elderly ICU patients in relation to premorbid functioning [9]. Here, we present the first large transnational study to show the influence of frailty on 30-day mortality in a large group of ICU patients of 80 years and above. We found that increased degree of frailty, being admitted to the ICU in a non-elective fashion and high SOFA score at admission (per point) were the three most important factors associated with ICU mortality or 30-day mortality.

Many previous studies on survival in VIPs are retrospective, usually single-centre and often small [5]. In these studies case mix differences profoundly affect outcome and ICU mortality ranges from $14 \%$ to $46 \%$ and hospital mortality from $28 \%$ to $48 \%[4,13]$. Unfortunately, fixed 30-day mortality is seldom reported since this information is often difficult to retrieve in many countries. Only two studies reported 30-day mortality and this was found to be $34.6 \%$ and $35 \%$, respectively $[14,15]$, which is similar to the overall 30 -day mortality of $32.4 \%$ in our study. The ICU mortality in our study of $22.1 \%$ and the 30 -day mortality of $32.4 \%$ are in the same range as those previously published in prospective cohort from Canada: $21.8 \%$ and $35 \%$, respectively [16]. However, in that study patients with an anticipated ICU length of stay less than $24 \mathrm{~h}$ were excluded. This may also explain the differences found in the LOS in these studies (median 4 days in the Canadian cohort versus 2.3 days in this study). Indeed, patients with a LOS less than $24 \mathrm{~h}$ were significantly more often admitted after planned surgery (see ESM Table 3). A striking finding was that ICU mortality was found to be higher in patients with shorter $(<24 \mathrm{~h})$ ICU stay compared with longer stay. A potential explanation for this finding could be that many patients with uncertain benefit from intensive care are admitted for "in-ICU triage" and, if found to be non-responsive regarding vital functions stabilization, treatment was stopped early.

We recruited 5132 very old intensive care patients from 21 European countries and found presence of frailty to be independently associated with 30-day mortality. Frailty has been defined as a clinical state of increased vulnerability from age-associated decline in physiological reserves and function in a wide range of physiological systems [2]. This concept of frailty has been used by geriatricians showing an association with adverse outcomes including decline in functional performance, prolonged LOS, institutionalisation, and mortality. The concept of frailty has recently spread to other areas of medicine $[17$, 18] and has been introduced as a potentially useful concept in (elderly) ICU patients [19]. Using a frailty index, one study found a strong association between increased frailty and 90-day mortality [20]. In a Canadian study, a lower index on a frailty scale was independently associated with increased survival in 610 patients followed for 12 months $[4,21]$. A recent multicentre American study found an association between greater degree of frailty, measured with the Clinical Frailty Scale, and higher risk with increasing frailty on 3- and 12-month mortality [22]. That study did not specifically report results in the very old, but found frailty to be common, even in younger ICU patients. In our study, we found an association between 
Table 3 Survival analysis all patients (Cox model: all patients are censored at a maximum of $\mathbf{3 0}$ days)

\begin{tabular}{|c|c|c|}
\hline & $\mathrm{HR}(95 \% \mathrm{Cl})$ & $p$ \\
\hline \multicolumn{3}{|l|}{ Univariate analysis } \\
\hline \multicolumn{3}{|l|}{ Frailty } \\
\hline Vulnerable (4) vs fit (1-3) & $1.24(1.07-1.43)$ & 0.005 \\
\hline Frail (5-9) vs fit (1-3) & $1.88(1.67-2.1)$ & $<0.001$ \\
\hline \multicolumn{3}{|l|}{ Frailty } \\
\hline 2 vs 1 & $1.33(0.91-1.93)$ & 0.136 \\
\hline 3 vs 1 & $1.27(0.89-1.81)$ & 0.187 \\
\hline 4 vs 1 & $1.56(1.09-2.23)$ & 0.014 \\
\hline 5 vs 1 & $1.97(1.38-2.81)$ & $<0.001$ \\
\hline 6 vs 1 & $2.16(1.51-3.08)$ & $<0.001$ \\
\hline 7 vs 1 & $3.08(2.15-4.41)$ & $<0.001$ \\
\hline 8 vs 1 & $3.29(2.20-4.92)$ & $<0.001$ \\
\hline 9 vs 1 & $4.50(2.45-8.25)$ & $<0.001$ \\
\hline \multicolumn{3}{|l|}{ Age } \\
\hline 5-year increase & $1.21(1.14-1.30)$ & $<0.001$ \\
\hline \multicolumn{3}{|l|}{ Gender } \\
\hline Male vs female & $1.11(1.01-1.22)$ & 0.039 \\
\hline \multicolumn{3}{|l|}{ SOFA score } \\
\hline One-point increase & $1.14(1.13-1.16)$ & $<0.001$ \\
\hline \multicolumn{3}{|l|}{ Type of admission } \\
\hline Acute vs elective & $6.72(5.22-8.67)$ & $<0.001$ \\
\hline \multicolumn{3}{|l|}{ Multivariate analysis } \\
\hline \multicolumn{3}{|l|}{ Frailty } \\
\hline Vulnerable (4) vs fit (1-3) & $1.19(1.03-1.38)$ & 0.021 \\
\hline Frail (5-9) vs fit (1-3) & $1.54(1.38-1.73)$ & $<0.001$ \\
\hline \multicolumn{3}{|l|}{ Age } \\
\hline 5-year increase & $1.2(1.12-1.28)$ & $<0.001$ \\
\hline \multicolumn{3}{|l|}{ Gender } \\
\hline Male vs female & $1.17(1.06-1.29)$ & 0.002 \\
\hline \multicolumn{3}{|l|}{ SOFA score } \\
\hline One-point increase & $1.13(1.12-1.14)$ & $<0.001$ \\
\hline \multicolumn{3}{|l|}{ Type of admission } \\
\hline Acute vs elective & $4.72(3.65-6.10)$ & $<0.001$ \\
\hline \multicolumn{3}{|c|}{ Acute admissions only ( $n=4115)$} \\
\hline \multicolumn{3}{|l|}{ Frailty } \\
\hline Vulnerable (4) vs fit (1-3) & $1.18(1.02-1.38)$ & 0.031 \\
\hline Frail (5-9) vs fit (1-3) & $1.53(1.36-1.72)$ & $<0.001$ \\
\hline \multicolumn{3}{|l|}{ Age } \\
\hline 5-year increase & $1.19(1.12-1.28)$ & $<0.001$ \\
\hline \multicolumn{3}{|l|}{ Gender } \\
\hline Male vs female & $1.17(1.06-1.29)$ & 0.002 \\
\hline \multicolumn{3}{|l|}{ SOFA score } \\
\hline One-point increase & $1.13(1.12-1.14)$ & $<0.001$ \\
\hline
\end{tabular}


Table 3 continued

\begin{tabular}{lllr}
\hline Survival estimates & $\%(95 \% \mathrm{Cl})$ & & At 30 days \\
\cline { 2 - 4 } & At 1 day & At 3 days & $76 \%(74-78)$ \\
Fit & $95 \%(94-96)$ & $90 \%(89-92)$ & $71 \%(68-74)$ \\
Vulnerable & $95 \%(93-96)$ & $90 \%(88-92)$ & $59 \%(57-61)$ \\
Frail & $92 \%(91-93)$ & $85 \%(83-86)$ & \\
\hline
\end{tabular}

Outcome $=$ death in the first 30 days following ICU admission

$N=5021$ patients with available data for outcome, frailty, age, gender, SOFA score and type of ICU admission

30-day mortality and increased frailty using a slightly expanded nine-point frailty scale.

Survival at 30 days was, however, most affected by type of admission; acute admissions had the highest risk of dying. Obviously, this is the result of selection bias related to urgency of admission. Patients admitted after planned surgery are younger, less frail and have a lower SOFA score on the first day of admission than patients after emergency admission. This selection of "better" and more prepared patients leads to lower mortality rates. Vice versa, the highest mortality was found after trauma, with a mortality of nearly $50 \%$ after 30 days.

Other important factors that remained independently associated with 30-day survival after adjustment were the SOFA score, gender and age. Several severity of illness scores have been applied and shown to be associated with mortality in studies in a general (non-elderly) ICU population. However, none of these models was found sufficiently reliable to be used in the elderly ICU patients [23]. For example, in a prospective study of 1033 VIPs from Canadian ICUs, the researchers presented a risk score based on values present around ICU admission. A combination based on age, Glasgow Coma Score (GCS), serum creatinine and $\mathrm{pH}$ performed well with c-statistic of 0.72 [16]. However, risk prognostication in a critical ill elderly population is associated with other factors as well, factors more intimately associated with age like co-morbidity, activities of daily life, frailty, dementia and sarcopenia. Hence, severity scores that focus on physiological abnormalities will probably not perform better than the general prognostic scoring systems in this group. Although we found an association between frailty and short-term outcomes, frailty alone cannot predict such outcomes. Strikingly, we found an association between frailty and SOFA score measured within the first $24 \mathrm{~h}$ of admission (see Table 1). The patients with a higher frailty class had more SOFA points. We hypothesized that this is caused by comorbidities present prior to ICU admission that, once the patient is admitted and critically ill, translate or progress into more organ failure on the ICU. We think that this simple-to-use clinical frailty scale should be a natural candidate to include in a future composite risk score for (the very old) ICU patients.

Despite the rather short median ICU LOS, most of the VIPs in our study had a high admission SOFA score, and most received one or more organ support therapies. VIPs have previously been found to receive less active treatment than their younger counterparts. From France the daily workload on VIPs compared to a group aged 65-80 was found to be lower [24]; and a Norwegian study in elderly patients reported that mechanical ventilation is less often applied and with shorter duration than in an age group from 50 to 80 years: $40.6 \%$ vs $56.1 \%$ [25]. More recent data from Canada demonstrates higher treatment intensity and $72 \%$ of the VIPs received mechanical ventilation for a mean of 7.1 days [16]. Neither that study nor the present one has a comparison group, but both indicate an increased therapeutic approach in the VIPs.

The strength of this study is the high number of critically ill VIPs recruited prospectively during a limited time period (8 months), hence reducing the effects of time bias on approach and attitude to treatment. It is also the first large transnational study in this particular age group. We included all ICU admissions, not only those with certain admissions or certain LOS; hence the results should have an acceptable external validity. On the other hand, there are several limitations. Since we deliberately made the study rather simple, we missed certain details of the patients, like co-morbidity and duration of organ support. The proportion of the VIPs for all separate ICUs was not recorded. We also have no long-term survival and functional outcome data in survivors. This was, however, not within the scope of this study. The chosen way of measuring frailty (clinical frailty scale) is subjective and may have a higher inter-rater variability than more objective measures of frailty. However, given the hectic circumstances around an acute admission in very old patients, there will never be time for more detailed screening like grip strength and walking speed, both important for the concept of frailty. Additionally, the evaluation of such measures is not feasible in most of the patients and might reflect the effect of the acute illness rather than the pre-admission frailty status. 


\section{Conclusion}

A simple frailty screening tool such as the Clinical Frailty Scale adds value to the global assessment of VIPs, and frailty is a significant factor for reduced 30-day survival. However, we found that acute ICU admission had the strongest impact on survival, while age had a smaller impact. Future studies are required to clarify how we can include frailty and its significance in ICU predictive scores.

\section{Electronic supplementary material}

The online version of this article (doi:10.1007/s00134-017-4940-8) contains supplementary material, which is available to authorized users.

\begin{abstract}
Author details
${ }^{1}$ Department of Clinical Medicine, University of Bergen, Bergen, Norway. ${ }^{2}$ Department of Anaesthesia and Intensive Care, Haukeland University Hospital, Bergen, Norway. ${ }^{3}$ Department of Intensive Care Medicine, University Medical Center, University Utrecht, Utrecht, The Netherlands. ${ }^{4}$ Department of Rehabilitation Hospital Ancelle di Cremona, Cremona, Italy. ${ }^{5}$ Geriatric Research Group, Brescia, Italy. ${ }^{6}$ Department of Anaesthesia and Intensive Care, Ålesund Hospital, Ålesund, Norway. ${ }^{7}$ Department of Circulation and Medical Imaging, NTNU, Trondheim, Norway. ${ }^{8}$ Department of Intensive Care Medecine, CIBER Enfermedades Respiratorias, Corporacion Sanitaria Universitaria Parc Tauli, Autonomous University of Barcelona, Sabadell, Spain. ${ }^{9}$ Department of Anaesthesia and Intensive Care Medicine, Aarhus University Hospital, Aarhus, Denmark. ${ }^{10}$ Laboratorio di Epidemiologia Clinica, Centro di Coordinamento GiViTI Dipar timento di Salute Pubblica, IRCCS-Istituto di Ricerche Farmacologiche "Mario Negri", Villa Camozzi, 24020 Ranica (Bergamo), Italy. ${ }^{11}$ Unite de Recherche en Epidemiologie Systemes d'Information et Mode lisation U707, Institut national de la sante et de la recherche medicale, Paris, France. ${ }^{12}$ St George's University Hospital, London, UK. ${ }^{13}$ Grande Ospedale Metropolitano Niguarda, Milan, Italy. ${ }^{14}$ Department of Cardiology, Pulmonology and Angiology, University Hospital, Düsseldorf, Germany. ${ }^{15}$ Mater Misericordiae University Hospital, Dublin, Ireland. ${ }^{16}$ Unidade de Cuidados Intensivos Neurocríticos, Hospital de São José, Centro Hospitalar de Lisboa Central, Faculdade de Ciência Médicas de Lisboa, Nova Médical School, Lisbon, Portugal. ${ }^{17}$ Department of Intensive Care 1K12IC, Ghent University Hospital, Ghent, Belgium. ${ }^{18}$ Karolinska University Hospital, Solna, Sweden. ${ }^{19}$ Geneva University Hospitals, Geneva, Switzerland. ${ }^{20}$ Department of Intensive Care, University Medical Center, University Utrecht, Utrecht, The Netherlands. ${ }^{21}$ Intensive Care and Perioperative Medicine Division, Jagiellonian University Medical College, Kraków, Poland. ${ }^{22}$ Kardinal Schwarzenberg Hospital, Schwarzach, Austria.

${ }^{23}$ Intensive Care Unit General Hospital of Larissa Tsakalof, Larissa, Greece.

${ }^{24}$ Assistance Publique-Hôpitaux de Paris, Hôpital Saint-Antoine, service de réanimation médicale, Paris, France. ${ }^{25}$ Sorbonne Universités, UPMC Univ Paris 06, Institut Pierre Louis d'Epidémiologie et de Santé Publique, Paris, France. ${ }^{26}$ INSERM, Institut Pierre Louis d'Epidémiologie et de Santé Publique, Paris, France.
\end{abstract}

\section{Acknowledgements}

This study was endorsed by the ESICM. Free support for running the electronic database and eCRF was granted from the Department of Epidemiology, University of Aarhus, Denmark. The VIP1-study collaborators are listed in ESM Table 7.

Received: 11 August 2017 Accepted: 14 September 2017

Published online: 21 September 2017

\section{References}

1. Nguyen YL, Angus DA, Boumendil A, Guidet B (2011) The challenge of admitting the very elderly to intensive care. Ann Intensive Care 1:29. doi:10.1186/2110-5820-1-29
2. McDermid RC, Bagshaw SM (2014) Scratching the surface: the burden of frailty in critical care. Intensive Care Med 40:740-742. doi:10.1007/ s00134-014-3246-3

3. Muscedere J, Waters B, Varambally A, Bagshaw SM, Boyd JG, Maslove D et al (2017) The impact of frailty on intensive care unit outcomes: a systematic review and meta-analysis. Intensive Care Med 43:1105-1122. doi:10.1007/s00134-017-4867-0

4. Heyland D, Cook D, Bagshaw SM, Garland A, Stelfox HT, Mehta S et al (2015) The very elderly admitted to ICU: a quality finish? Crit Care Med 43:1352-1360. doi:10.1097/CCM.0000000000001024

5. Flaatten H, Garrouste-Orgeas M (2015) The very old ICU patient: a never-ending story. Intensive Care Med 41:1996-1998. doi:10.1007/ s00134-015-4052-2

6. Boumendil A, Angus DC, Guitonneau AL, Menn AM, Ginsburg C, Takun K, Davido A, Masmoudi R, Doumenc B, Pateron D, Garrouste-Orgeas M, Somme D, Simon T, Aegerter Ph, Guidet B (2012) Variability of intensive care admission decisions for the very elderly. PLoS One 7:e34387. doi:10.1371/journal.pone.0034387

7. Poole D, Finazzi S, Nattino G, Radrizzani D, Gristina G, Malacarne P, Livigni S, Bertolini G (2017) The prognostic importance of chronic end-stage diseases in geriatric patients admitted to 163 Italian ICUs. Minerva Anestesiol. doi:10.23736/S0375-9393.17.11919-X

8. Tabah A, Philippart F, Timsit JF, Willems V, Français A, Leplège A et al (2010) Quality of life in patients aged 80 or over after ICU discharge. Crit Care 14(1):R2. doi:10.1186/cc8231

9. Flaatten $\mathrm{H}$, de Lange DW, Artigas A, Bin D, Moreno R, Christensen S et al (2017) The status of intensive care medicine research and a future agenda for very old patients in the ICU. Intensive Care Med 43:1319-1328. doi:10.1007/s00134-017-4718-z

10. Vincent JL, Moreno R, Takala J, Willatts S, de Mendonça A, Bruining H et al (1996) The SOFA (Sepsis-related Organ Failure Assessment) score to describe organ dysfunction/failure. On behalf of the Working Group on Sepsis-Related Problems of the European Society of Intensive Care Medicine. Intensive Care Med 22:707-710

11. Rockwood K, Song X, MacKnight C, Bergman H, Hogan DB, McDowell I et al (2005) A global clinical measure of fitness and frailty in elderly people. CMAJ 173:489-495. doi:10.1503/cmaj.050051

12. Guidet B, Hodgson E, Feldman C, Paruk F, Lipman J, Koh Y, Vincent JL, Azoulay E, Sprung C (2014) The Durban World Congress Ethics Round Table conference report: II. Withholding or withdrawing of treatment in elderly patients admitted to the intensive care unit. J Crit Care 29:896-901. doi:10.1016/j.jcrc.2014.08.004

13. Roch A, Wiramus S, Pauly V, Forel J-M, Guervilly C, Gainnier M et al (2011) Long-term outcome in medical patients aged 80 or over following admission to an intensive care unit. Crit Care 15:R36. doi:10.1186/cc9984

14. Nielsson MS, Christiansen CF, Johansen MB, Rasmussen BS, Tønnesen E, Nørgaard M (2014) Mortality in elderly ICU patients: a cohort study. Acta Anaesthesiol Scand 58:19-26. doi:10.1111/aas.12211

15. Lown DJ, Knott J, Rechnitzer T, Maclsaac C (2013) Predicting short-term and long-term mortality in elderly emergency patients admitted for intensive care. Crit Care Resusc 15:49-55

16. Ball IM, Bagshaw SM, Burns KEA, Cook DJ, Day AG, Dodek PM et al (2016) A clinical prediction tool for hospital mortality in critically ill elderl patients. J Crit Care 35:206-212. doi:10.1016/j.jcrc.2016.05.026

17. Hall DE, Arya S, Schmid KK, Carlson MA, Lavedan P, Bailey TL et al (2017) Association of a frailty screening initiative with postoperative survival at 30, 180, and 365 days. JAMA Surg 152:233-240. doi:10.1001/ jamasurg.2016.4219

18. Afilalo J, Lauck S, Kim DH, Lefèvre T, Piazza N, Lachapelle K et al (2017) Frailty in older adults undergoing aortic valve replacement: the FRAILTYAVR study. J Am Coll Cardiol 70:689-700. doi:10.1016/j.jacc.2017.06.24

19. McDermid RC, Stelfox HT, Bagshaw SM (2011) Frailty in the critically ill: a novel concept. Crit Care 15:301. doi:10.1186/cc9297

20. Zeng A, Song X, Dong J, Mitnitski A, Liu J, Guo Z et al (2015) Mortality in relation to frailty in patients admitted to a specialized geriatric intensive care unit. J Gerontol A Biol Sci Med Sci 70:1586-1594. doi:10.1093/gerona/glv084

21. Heyland DK, Garland A, Bagshaw SM, Cook D, Rockwood K, Stelfox HT et al (2015) Recovery after critical illness in patients aged 80 years or older: a multi-center prospective observational cohort study. Intensive Care Med 41:1911-1920. doi:10.1007/s00134-015-4028-2 
22. Brummel NE, Bell SP, Girard TD, Pandharipande PP, Jackson JC, Morandi A et al (2017) Frailty and subsequent disability and mortality among patients with critical illness. Am J Respir Crit Care Med 196:64-72. doi:10.1164/rccm.201605-09390C

23. Minne L, Ludikhuize J, de Jonge E, de Rooij S, Abu-Hanna A (2011) Prognostic models for predicting mortality in elderly ICU patients: a systematic review. Intensive Care Med 37:1258-1268. doi:10.1186/cc7160
24. Boumendil A, Aegerter P, Guidet B, CUB-Rea Network (2005) Treatment intensity and outcome of patients aged 80 and older in intensive care units: a multicenter matched-cohort study. J Am Geriatr Soc 53:88-93. doi:10.1111/j.1532-5415.2005.53016.x

25. Andersen FH, Kvåle R (2012) Do elderly intensive care unit patients receive less intensive care treatment and have higher mortality? Acta Anaesthesiol Scand 56:1298-1305. doi:10.1111/j.1399-6576.2012.02782.x 